\title{
ON THE SINE INTEGRAL AND THE CONVOLUTION
}

\section{BRIAN FISHER and FATMA AL-SIREHY}

Received 7 June 2001

The sine integral $\operatorname{Si}(\lambda x)$ and the cosine integral $\operatorname{Ci}(\lambda x)$ and their associated functions $\mathrm{Si}_{+}(\lambda x), \mathrm{Si}_{-}(\lambda x), \mathrm{Ci}_{+}(\lambda x), \mathrm{Ci}_{-}(\lambda x)$ are defined as locally summable functions on the real line. Some convolutions of these functions and $\sin (\mu x), \sin _{+}(\mu x)$, and $\sin _{-}(\mu x)$ are found. 2000 Mathematics Subject Classification: 33B10, 46F10.

The sine integral $\operatorname{Si}(x)$ is defined by

$$
\int_{0}^{x} u^{-1} \sin u d u
$$

(see Sneddon [6]). This integral is convergent for all $x$. More generally, for all $\lambda \neq 0$, we define $\operatorname{Si}(\lambda x)$ by

$$
\operatorname{Si}(\lambda x)=\int_{0}^{\lambda x} u^{-1} \sin u d u=\int_{0}^{x} u^{-1} \sin (\lambda u) d u
$$

and we define $\mathrm{Si}_{+}(\lambda x)$ and $\mathrm{Si}_{-}(\lambda x)$ by

$$
\operatorname{Si}_{+}(\lambda x)=H(x) \operatorname{Si}(\lambda x), \quad \operatorname{Si}_{-}(\lambda x)=H(-x) \operatorname{Si}(\lambda x),
$$

(see [1]).

It is easily proved that

$$
\left[\mathrm{Si}_{+}(\lambda x)\right]^{\prime}=\sin (\lambda x) x_{+}^{-1}
$$

We need the following lemma which was proved in [1].

LEMMA 1. If $\lambda \neq 0$, then

$$
\int_{0}^{\infty} u^{-1} \sin (\lambda u) d u=\frac{1}{2} \operatorname{sgn} \lambda \cdot \pi
$$

The cosine integral $\mathrm{Ci}(x)$ is defined for $x>0$ by

$$
\mathrm{Ci}(x)=-\int_{x}^{\infty} u^{-1} \cos u d u
$$

(see Sneddon [6]). This integral is divergent for $x \leq 0$; but in [3], $\mathrm{Ci}(\lambda x)$ was defined as a locally summable function on the real line by

$$
\operatorname{Ci}(\lambda x)=-\int_{\lambda x}^{\infty} u^{-1}[\cos u-H(1-u)] d u+H(1-\lambda x) \ln |\lambda x|,
$$


where $H$ denotes Heaviside's function. In particular,

$$
\mathrm{Ci}(x)=-\int_{x}^{\infty} u^{-1}[\cos u-H(1-u)] d u+H(1-x) \ln |x| .
$$

It was proved in [4] that the cosine integral is an even function. We can therefore define $\mathrm{Ci}(\lambda x)$ by

$$
\operatorname{Ci}(\lambda x)=-\int_{|\lambda x|}^{\infty} u^{-1} \cos u d u=-\int_{|x|}^{\infty} u^{-1} \cos (\lambda u) d u, \quad \lambda, x \neq 0,
$$

simplifying the definition given in [3].

The locally summable functions $\mathrm{Ci}_{+}(\lambda x)$ and $\mathrm{Ci}_{-}(\lambda x)$ are now defined for $\lambda \neq 0$ by

$$
\mathrm{Ci}_{+}(\lambda x)=H(x) \operatorname{Ci}(\lambda x), \quad \mathrm{Ci}_{-}(\lambda x)=H(-x) \operatorname{Ci}(\lambda x) .
$$

It was proved in [3] that

$$
\left[\mathrm{Ci}_{+}(\lambda x)\right]^{\prime}=\cos (\lambda x) x_{+}^{-1}-(c-\ln |\lambda|) \delta(x),
$$

where

$$
c=\int_{0}^{\infty} u^{-1}[\cos u-H(1-u)] d u
$$

We also need the following lemma which was also proved in [4].

LEMMA 2. If $x>0$, then

$$
\int_{0}^{x} u^{-1}[\cos (\lambda u)-1] d u=c+\operatorname{Ci}(\lambda x)-\ln |\lambda x| .
$$

The classical definition of the convolution of two functions $f$ and $g$ is as follows.

Definition 3. Let $f$ and $g$ be functions. Then the convolution $f * g$ is defined by

$$
(f * g)(x)=\int_{-\infty}^{\infty} f(t) g(x-t) d t
$$

for all points $x$ for which the integral exists.

It follows easily from the definition that if $f * g$ exists then $g * f$ exists and

$$
f * g=g * f
$$

and if $(f * g)^{\prime}$ and $f * g^{\prime}$ (or $f^{\prime} * g$ ) exists, then

$$
(f * g)^{\prime}=f * g^{\prime}\left(\text { or } f^{\prime} * g\right) .
$$

Definition 3 can be extended to define the convolution $f * g$ of two distributions $f$ and $g$ in $\mathscr{D}^{\prime}$ with the following definition, see Gel'fand and Shilov [5]. 
DefinItion 4. Let $f$ and $g$ be distributions in $\mathscr{D}^{\prime}$. Then the convolution $f * g$ is defined by the equation

$$
\langle(f * g)(x), \phi\rangle=\langle f(y),\langle g(x), \phi(x+y)\rangle\rangle
$$

for arbitrary $\phi$ in $\mathscr{D}$, provided that $f$ and $g$ satisfy either of the following conditions:

(a) either $f$ or $g$ has bounded support,

(b) the supports of $f$ and $g$ are bounded on the same side.

It follows that if the convolution $f * g$ exists by this definition then (15) and (16) are satisfied.

In the following, the locally summable functions $\sin _{ \pm}(\lambda x)$ and $\cos _{ \pm}(\lambda x)$ are defined by

$$
\begin{array}{ll}
\sin _{+}(\lambda x)=H(x) \sin (\lambda x), & \sin _{-}(\lambda x)=H(-x) \sin (\lambda x), \\
\cos _{+}(\lambda x)=H(x) \cos (\lambda x), & \cos _{-}(\lambda x)=H(-x) \cos (\lambda x) .
\end{array}
$$

THEOREM 5. If $\lambda, \mu \neq 0$, then the convolution $\operatorname{Si}_{+}(\lambda x) * \sin _{+}(\mu x)$ exists and

$$
\begin{aligned}
\mathrm{Si}_{+}(\lambda x) * \sin _{+}(\mu x)= & -\frac{1}{2} \mu^{-1} \sin (\mu x)\left\{\mathrm{Ci}_{+}[(\lambda-\mu) x]-\mathrm{Ci}_{+}[(\lambda+\mu) x]\right\} \\
& +\mu^{-1} \mathrm{Si}_{+}(\lambda x)-\frac{1}{2} \mu^{-1} \cos (\mu x)\left\{\mathrm{Si}_{+}[(\lambda-\mu) x]+\mathrm{Si}_{+}[(\lambda+\mu) x]\right\} \\
& -\frac{1}{2} \mu^{-1} \ln \left|\frac{\lambda+\mu}{\lambda-\mu}\right| \sin _{+}(\mu x)
\end{aligned}
$$

if $\lambda \neq \pm \mu$; and

$$
\begin{aligned}
\mathrm{Si}_{+}(\lambda x) * \sin _{+}(\lambda x)= & \lambda^{-1} \mathrm{Si}_{+}(\lambda x)-\frac{1}{2} \lambda^{-1}\left[\ln \left|\frac{1}{2} \lambda\right|-c\right] \sin _{+}(\lambda x) \\
& -\frac{1}{2} \lambda^{-1} \cos (\lambda x) \mathrm{Si}_{+}(2 \lambda x) \\
& +\frac{1}{2} \lambda^{-1} \sin (\lambda x)\left[\mathrm{Ci}_{+}(2 \lambda x)-\ln x_{+}\right]
\end{aligned}
$$

if $\lambda= \pm \mu$.

Proof. It is obvious that $\operatorname{Si}_{+}(\lambda x) * \sin _{+}(\mu x)=0$ if $x<0$. If $x>0$ and $\lambda \neq \pm \mu$, we have

$$
\begin{aligned}
\operatorname{Si}_{+}(\lambda x) * \sin _{+}(\mu x) & =\int_{0}^{x} \sin [\mu(x-t)] \int_{0}^{t} u^{-1} \sin (\lambda u) d u d t \\
& =\int_{0}^{x} u^{-1} \sin (\lambda u) \int_{u}^{x} \sin [\mu(x-t)] d t d u \\
& =\mu^{-1} \int_{0}^{x} u^{-1} \sin (\lambda u)\{1-\cos [\mu(x-u)]\} d u \\
& =\mu^{-1} \operatorname{Si}(\lambda x)-\mu^{-1} I,
\end{aligned}
$$


where

$$
\begin{aligned}
I= & \int_{0}^{x} u^{-1} \sin (\lambda u) \cos [\mu(x-u)] d u \\
= & \cos (\mu x) \int_{0}^{x} u^{-1} \sin (\lambda u) \cos (\mu u) d u+\sin (\mu x) \int_{0}^{x} u^{-1} \sin (\lambda u) \sin (\mu u) d u \\
= & \frac{1}{2} \cos (\mu x) \int_{0}^{x} u^{-1}\{\sin [(\lambda-\mu) u]+\sin [(\lambda+\mu) u]\} d u \\
& +\frac{1}{2} \sin (\mu x) \int_{0}^{x} u^{-1}\{\cos [(\lambda-\mu) u]-\cos [(\lambda+\mu) u]\} d u \\
= & \frac{1}{2} \cos (\mu x)\{\operatorname{Si}[(\lambda-\mu) x]+\operatorname{Si}[(\lambda+\mu) x]\} \\
& +\frac{1}{2} \sin (\mu x)\left\{\operatorname{Ci}[(\lambda-\mu) x]-\operatorname{Ci}[(\lambda+\mu) x]+\ln \left|\frac{\lambda+\mu}{\lambda-\mu}\right|\right\}
\end{aligned}
$$

on using Lemma 2; and (19) follows from (22) and (23).

If $\lambda= \pm \mu$, (21) is replaced by

$$
\begin{aligned}
\operatorname{Si}_{+}(\lambda x) * \sin _{+}(\lambda x) & =\lambda^{-1} \int_{0}^{x} u^{-1} \sin (\lambda u)\{1-\cos [\lambda(x-u)]\} d u \\
& =\lambda^{-1} \operatorname{Si}(\lambda x)-\lambda^{-1} J
\end{aligned}
$$

where

$$
\begin{aligned}
J & =\int_{0}^{x} u^{-1} \sin (\lambda u) \cos [\lambda(x-u)] d u \\
& =\frac{1}{2} \cos (\lambda x) \int_{0}^{x} u^{-1} \sin (2 \lambda u) d u-\frac{1}{2} \sin (\lambda x) \int_{0}^{x} u^{-1}[\cos (2 \lambda u)-1] d u \\
& =\frac{1}{2} \cos (\lambda x) \operatorname{Si}(2 \lambda x)+\frac{1}{2}[\ln |2 \lambda x|-c] \sin (\lambda x)-\frac{1}{2} \sin (\lambda x) \operatorname{Ci}(2 \lambda x)
\end{aligned}
$$

on using Lemma 2; and (20) follows from (24) and (25).

COROLLARY 6. If $\lambda, \mu \neq 0$, then the convolution $\mathrm{Si}_{+}(\lambda x) * \cos _{+}(\mu x)$ exists and

$$
\begin{aligned}
\mathrm{Si}_{+}(\lambda x) * \cos _{+}(\mu x)= & \frac{1}{2} \mu^{-1} \sin (\mu x)\left\{\mathrm{Si}_{+}[(\lambda-\mu) x]+\mathrm{Si}_{+}[(\lambda+\mu) x]\right\} \\
& -\frac{1}{2} \mu^{-1} \cos (\mu x)\left\{\mathrm{Ci}_{+}[(\lambda-\mu) x]-\mathrm{Ci}_{+}[(\lambda+\mu) x]\right\} \\
& -\frac{1}{2} \mu^{-1} \ln \left|\frac{\lambda+\mu}{\lambda-\mu}\right| \cos _{+}(\mu x)
\end{aligned}
$$

if $\lambda \neq \pm \mu$; and

$$
\begin{aligned}
\mathrm{Si}_{+}(\lambda x) * \cos _{+}(\lambda x)= & \frac{1}{2} \lambda^{-1} \sin (\lambda x) \mathrm{Si}_{+}(2 \lambda x)-\frac{1}{2} \lambda^{-1}\left[\ln \left|\frac{1}{2} \lambda\right|-c\right] \cos _{+}(\lambda x) \\
& +\frac{1}{2} \lambda^{-1} \cos (\lambda x)\left[\mathrm{Ci}_{+}(2 \lambda x)-\ln x_{+}\right]
\end{aligned}
$$

if $\lambda= \pm \mu$. 
Proof. It follows from (4), (11), (16), and (19) that

$$
\begin{aligned}
{\left[\mathrm{Si}_{+}(\lambda x) * \sin _{+}(\mu x)\right]^{\prime}=} & \mu \mathrm{Si}_{+}(\lambda x) * \cos _{+}(\mu x) \\
= & \mu^{-1} \sin (\lambda x) x_{+}^{-1}-\frac{1}{2} \ln \left|\frac{\lambda+\mu}{\lambda-\mu}\right| \cos _{+}(\mu x) \\
& -\frac{1}{2} \cos (\mu x)\left\{\mathrm{Ci}_{+}[(\lambda-\mu) x]-\mathrm{Ci}_{+}[(\lambda+\mu) x]\right\} \\
& -\frac{1}{2} \mu^{-1} \sin (\mu x)\{\cos [(\lambda-\mu) x]-\cos [(\lambda+\mu) x]\} x_{+}^{-1} \\
& -\frac{1}{2} \mu^{-1} \ln \left|\frac{\lambda+\mu}{\lambda-\mu}\right| \sin (\mu x) \delta(x) \\
& +\frac{1}{2} \sin (\mu x)\left\{\mathrm{Si}_{+}[(\lambda-\mu) x]+\mathrm{Si}_{+}[(\lambda+\mu) x]\right\} \\
& -\frac{1}{2} \mu^{-1} \cos (\mu x)\{\sin [(\lambda+\mu) x]+\sin [(\lambda-\mu) x]\} x_{+}^{-1} \\
= & -\frac{1}{2} \ln \left|\frac{\lambda+\mu}{\lambda-\mu}\right| \cos _{+}(\mu x) \\
& -\frac{1}{2} \cos (\mu x)\left\{\mathrm{Ci}_{+}[(\lambda+\mu) x]-\mathrm{Ci}_{+}[(\lambda-\mu) x]\right\} \\
& +\frac{1}{2} \sin (\mu x)\left\{\mathrm{Si}_{+}[(\lambda+\mu) x]+\mathrm{Si}_{+}[(\lambda-\mu) x]\right\}
\end{aligned}
$$

and (26) follows.

If $\lambda= \pm \mu$, it follows from (4), (11), (16), and (20) that

$$
\begin{aligned}
\lambda \mathrm{Si}_{+}(\lambda x) * \cos _{+}(\lambda x)= & \lambda^{-1} \sin (\lambda x) x_{+}^{-1}-\frac{1}{2}\left[\ln \left|\frac{1}{2} \lambda\right|-c\right] \cos _{+}(\lambda x) \\
& +\frac{1}{2} \sin (\lambda x) \mathrm{Si}_{+}(2 \lambda x)-\frac{1}{2} \lambda^{-1} \cos (\lambda x) \sin (2 \lambda x) x_{+}^{-1} \\
& +\frac{1}{2} \cos (\lambda x)\left[\mathrm{Ci}_{+}(2 \lambda x)-\ln x_{+}\right] \\
& +\frac{1}{2} \lambda^{-1} \sin (\lambda x)[\cos (2 \lambda x)-1] x_{+}^{-1} \\
= & -\frac{1}{2}\left[\ln \left|\frac{1}{2} \lambda\right|-c\right] \cos _{+}(\lambda x)+\frac{1}{2} \sin (\lambda x) \mathrm{Si}_{+}(2 \lambda x) \\
& +\frac{1}{2} \cos (\lambda x)\left[\mathrm{Ci}_{+}(2 \lambda x)-\ln x_{+}\right]
\end{aligned}
$$

and (27) follows.

COROLlary 7. If $\lambda, \mu \neq 0$, then the convolutions $\mathrm{Si}_{-}(\lambda x) * \sin _{-}(\mu x)$ and $\mathrm{Si}_{-}(\lambda x) *$ $\cos _{-}(\mu x)$ exist and

$$
\begin{aligned}
\mathrm{Si}_{-}(\lambda x) * \sin { }_{-}(\mu x)= & \frac{1}{2} \mu^{-1} \sin (\mu x)\left\{\mathrm{Ci}_{-}[(\lambda-\mu) x]-\mathrm{Ci}_{-}[(\lambda+\mu) x]\right\} \\
& -\mu^{-1} \mathrm{Si}_{-}(\lambda x)+\frac{1}{2} \mu^{-1} \operatorname{Cos}(\mu x)\left\{\mathrm{Si}_{-}[(\lambda-\mu) x]+\mathrm{Si}_{-}[(\lambda+\mu) x]\right\} \\
& +\frac{1}{2} \mu^{-1} \ln \left|\frac{\lambda+\mu}{\lambda-\mu}\right| \sin _{-}(\mu x),
\end{aligned}
$$




$$
\begin{aligned}
\mathrm{Si}_{-}(\lambda x) * \operatorname{Cos}_{-}(\mu x)= & -\frac{1}{2} \mu^{-1} \sin (\mu x)\left\{\mathrm{Si}_{-}[(\lambda-\mu) x]+\mathrm{Si}_{-}[(\lambda+\mu) x]\right\} \\
& +\frac{1}{2} \mu^{-1} \cos (\mu x)\left\{\mathrm{Ci}_{-}[(\lambda-\mu) x]-\mathrm{Ci}_{-}[(\lambda+\mu) x]\right\} \\
& +\frac{1}{2} \mu^{-1} \ln \left|\frac{\lambda+\mu}{\lambda-\mu}\right| \sin _{-}(\mu x)
\end{aligned}
$$

if $\lambda \neq \pm \mu$; and

$$
\begin{aligned}
& \mathrm{Si}_{-}(\lambda x) * \sin _{-}(\lambda x)=-\lambda^{-1} \mathrm{Si}_{-}(\lambda x)+\frac{1}{2} \lambda^{-1}\left[\ln \left|\frac{1}{2} \lambda\right|-c\right] \sin { }_{-}(\lambda x) \\
&+\frac{1}{2} \lambda^{-1} \cos (\lambda x) \mathrm{Si}_{-}(2 \lambda x) \\
&-\frac{1}{2} \lambda^{-1} \sin (\lambda x)\left[\mathrm{Ci}_{-}(2 \lambda x)-\ln x_{-}\right], \\
& \mathrm{Si}_{-}(\lambda x) * \cos _{-}(\lambda x)=-\frac{1}{2} \lambda^{-1} \sin (\lambda x) \mathrm{Si}_{-}(2 \lambda x)+\frac{1}{2} \lambda^{-1}\left[\ln \left|\frac{1}{2} \lambda\right|-c\right] \cos _{-}(\lambda x) \\
&-\frac{1}{2} \lambda^{-1} \cos (\lambda x)\left[\mathrm{Ci}_{-}(2 \lambda x)-\ln x_{-}\right] \\
& \text {if } \lambda= \pm \mu .
\end{aligned}
$$

Proof. Equations (30) and (31) follow on replacing $x$ by $-x$ in (19) and (26), respectively. Equations (32) and (33) follow on replacing $x$ by $-x$ in (20) and (27), respectively.

Definition 4 of the convolution is rather restrictive and so a neutrix convolution was introduced in [2]. In order to define the neutrix convolution we, first of all, let $\tau$ be a function in $\mathscr{D}$ satisfying the following properties:

(i) $\tau(x)=\tau(-x)$,

(ii) $0 \leq \tau(x) \leq 1$,

(iii) $T(x)=1$ for $|x| \leq 1 / 2$,

(iv) $\tau(x)=0$ for $|x| \geq 1$.

The function $\tau_{v}$ is now defined by

$$
\tau_{v}(x)= \begin{cases}1, & |x| \leq v, \\ \tau\left(v^{v} x-v^{v+1}\right), & x>v, \\ \tau\left(v^{v} x+v^{v+1}\right), & x<-v\end{cases}
$$

for $v>0$.

The following definition of the neutrix convolution was given in [2].

DEFINITION 8. Let $f$ and $g$ be distributions in $\mathscr{D}^{\prime}$ and let $f_{v}=f \tau_{v}$ for $v>0$. Then the neutrix convolution $f \circledast g$ is defined as the neutrix limit of the sequence $\left\{f_{v} * g\right\}$, provided that the limit $h$ exists in the sense that

$$
\mathrm{N}-\lim _{v \rightarrow \infty}\left\langle f_{v} * g, \varphi\right\rangle=\langle h, \varphi\rangle,
$$


for all $\varphi$ in $\mathscr{D}$, where $N$ is the neutrix (see van der Corput [7]), having domain $N^{\prime}$ the positive reals, range $N^{\prime \prime}$ the real numbers and with negligible functions finite linear sums of the functions

$$
v^{\lambda} \ln ^{r-1} v, \quad \ln ^{r} v, \quad(\lambda \neq 0, r=1,2, \ldots)
$$

and all functions which converge to zero in the usual sense as $v$ tends to infinity.

Note that in this definition, the convolution $f_{v} * g$ is defined in Gel'fand's and Shilov's sense, the distribution $f_{v}$ having bounded support.

It is easily seen that any results proved with the original definition hold with the new definition. The following theorem (proved in [2]) therefore holds, showing that the neutrix convolution is a generalization of the convolution.

THEOREM 9. Let $f$ and $g$ be distributions in $\mathscr{D}^{\prime}$ satisfying either condition (a) or condition (b) of Definition 4 (Gel'fand's and Shilov's [5]). Then the neutrix convolution $f \circledast g$ exists and

$$
f \circledast g=f * g .
$$

The next theorem was also proved in [2].

THEOREM 10. Let $f$ and $g$ be distributions in $\mathscr{D}^{\prime}$ and suppose that the neutrix convolution $f \circledast g$ exists. Then the neutrix convolution $f \circledast g^{\prime}$ exists and

$$
(f \circledast g)^{\prime}=f \circledast g^{\prime} .
$$

Note, however, that the neutrix convolution $(f \circledast g)^{\prime}$ is not necessarily equal to $f^{\prime} \circledast g$.

We now increase the set of negligible functions given here to include finite linear sums of the functions

$$
\cos (\lambda v), \quad \sin (\lambda v), \quad(\lambda \neq 0) .
$$

THEOREM 11. If $\lambda, \mu \neq 0$, then the neutrix convolution $\mathrm{Si}_{+}(\lambda x) \circledast \sin (\mu x)$ exists and

$$
\begin{aligned}
\mathrm{Si}_{+}(\lambda x) \circledast \sin (\mu x)= & -\frac{1}{4} \mu^{-1}[\operatorname{sgn}(\lambda+\mu)+\operatorname{sgn}(\lambda-\mu)] \pi \cos (\mu x) \\
& -\frac{1}{2} \mu^{-1} \ln \left|\frac{\lambda+\mu}{\lambda-\mu}\right| \sin (\mu x)
\end{aligned}
$$

if $\lambda \neq \pm \mu$; and

$$
\begin{aligned}
\mathrm{Si}_{+}(\lambda x) \circledast \sin (\lambda x)= & -\frac{1}{4} \lambda^{-1} \operatorname{sgn} \lambda \cdot \pi \cos (\lambda x) \\
& +\frac{1}{2} \lambda^{-1}[c-\ln |2 \lambda|] \sin (\lambda x)
\end{aligned}
$$

if $\lambda= \pm \mu$. 
Proof. We put $\left[\mathrm{Si}_{+}(\lambda x)\right]_{v}=\mathrm{Si}_{+}(\lambda x) \tau_{v}(x)$. Then the convolution $\left[\mathrm{Si}_{+}(\lambda x)\right]_{v} *$ $\sin (\mu x)$ exists by Definition 3 and

$$
\begin{aligned}
{\left[\mathrm{Si}_{+}(\lambda x)\right]_{v} * \sin (\mu x)=} & \int_{0}^{v} \mathrm{Si}_{+}(\lambda t) \sin [\mu(x-t)] d t \\
& +\int_{v}^{v+v^{-v}} \operatorname{Si}_{+}(\lambda t) \sin [\mu(x-t)] \tau_{v}(t) d t \\
= & I_{1}+I_{2},
\end{aligned}
$$

where it is easily seen that

$$
\lim _{n \rightarrow \infty} I_{2}=0 .
$$

Further,

$$
\begin{aligned}
I_{1}= & \int_{0}^{v} u^{-1} \sin (\lambda u) \int_{u}^{v} \sin [\mu(x-t)] d t d u \\
= & \mu^{-1} \int_{0}^{v} u^{-1} \sin (\lambda u)\{\cos [\mu(x-v)]-\cos [\mu(x-u)]\} d u \\
= & \mu^{-1} \cos [\mu(x-v)] \operatorname{Si}(\lambda v) \\
& -\frac{1}{2} \mu^{-1} \cos (\mu x) \int_{0}^{v} u^{-1}\{\sin [(\lambda+\mu) u]+\sin [(\lambda-\mu) u]\} d u \\
& +\frac{1}{2} \mu^{-1} \sin (\mu x) \int_{0}^{v} u^{-1}\{\cos [(\lambda+\mu) u]-\cos [(\lambda-\mu) u]\} d u \\
= & \mu^{-1} \cos [\mu(x-v)] \operatorname{Si}(\lambda v)-\frac{1}{2} \mu^{-1} \cos (\mu x)\{\operatorname{Si}[(\lambda+\mu) v]+\operatorname{Si}[(\lambda-\mu) v]\} \\
& +\frac{1}{2} \mu^{-1} \sin (\mu x)\left\{\operatorname{Ci}[(\lambda+\mu) v]-\operatorname{Ci}[(\lambda-\mu) v]-\ln \left|\frac{\lambda+\mu}{\lambda-\mu}\right|\right\}
\end{aligned}
$$

on using Lemma 2. It follows that

$$
\mathrm{N}_{v \rightarrow \infty} \lim _{1}=-\frac{1}{4} \mu^{-1}[\operatorname{sgn}(\lambda+\mu)+\operatorname{sgn}(\lambda-\mu)] \pi \cos (\mu x)-\frac{1}{2} \mu^{-1} \ln \left|\frac{\lambda+\mu}{\lambda-\mu}\right| \sin (\mu x)
$$

on using Lemma 1. Equation (40) now follows from (42), (43), and (45) .

If $\lambda= \pm \mu$, we have

$$
\begin{aligned}
{\left[\operatorname{Si}_{+}(\lambda x)\right]_{v} * \sin (\lambda x)=} & \int_{0}^{v} \operatorname{Si}_{+}(\lambda t) \sin [\lambda(x-t)] d t \\
& +\int_{v}^{v+v^{-v}} \operatorname{Si}_{+}(\lambda t) \sin [\lambda(x-t)] \tau_{v}(t) d t \\
= & J_{1}+J_{2},
\end{aligned}
$$


where it is easily seen that

$$
\lim _{n \rightarrow \infty} J_{2}=0
$$

Further,

$$
\begin{aligned}
J_{1}= & \int_{0}^{v} u^{-1} \sin (\lambda u) \int_{u}^{v} \sin [\lambda(x-t)] d t d u \\
= & \lambda^{-1} \int_{0}^{v} u^{-1} \sin (\lambda u)\{\cos [\lambda(x-v)]-\cos [\lambda(x-u)]\} d u \\
= & \lambda^{-1} \cos [\lambda(x-v)] \operatorname{Si}(\lambda v) \\
& -\frac{1}{2} \lambda^{-1} \cos (\lambda x) \int_{0}^{v} u^{-1} \sin (2 \lambda u) d u \\
& +\frac{1}{2} \lambda^{-1} \sin (\lambda x) \int_{0}^{v} u^{-1}[\cos (2 \lambda u)-1] d u \\
= & \lambda^{-1} \cos [\lambda(x-v)] \operatorname{Si}(\lambda v)-\frac{1}{2} \lambda^{-1} \cos (\lambda x) \operatorname{Si}(2 \lambda v) \\
& +\frac{1}{2} \lambda^{-1} \sin (\lambda x)[c+\operatorname{Ci}(2 \lambda v)-\ln |2 \lambda v|]
\end{aligned}
$$

on using Lemma 2. It follows that

$$
\mathrm{N}_{v \rightarrow \infty} \lim _{1}=-\frac{1}{4} \lambda^{-1} \operatorname{sgn} \lambda \cdot \pi \cos (\lambda x)+\frac{1}{2} \lambda^{-1}[c-\ln |2 \lambda|] \sin (\lambda x)
$$

on using Lemma 1. Equation (41) now follows from (46), (47), and (49).

COROLlary 12. If $\lambda, \mu \neq 0$, then the neutrix convolution $\operatorname{Si}_{+}(\lambda x) \circledast \cos (\mu x)$ exists and

$$
\begin{aligned}
\operatorname{Si}_{+}(\lambda x) \circledast \cos (\mu x)= & \frac{1}{4} \pi \mu^{-1}[\operatorname{sgn}(\lambda+\mu)+\operatorname{sgn}(\lambda-\mu)] \sin (\mu x) \\
& -\frac{1}{2} \mu^{-1} \ln \left|\frac{\lambda+\mu}{\lambda-\mu}\right| \cos (\mu x)
\end{aligned}
$$

if $\lambda \neq \pm \mu$; and

$$
\mathrm{Si}_{+}(\lambda x) \circledast \cos (\lambda x)=\frac{1}{4} \lambda^{-1} \operatorname{sgn} \lambda \cdot \pi \sin (\lambda x)+\frac{1}{2} \lambda^{-1}[c-\ln |2 \lambda|] \cos (\lambda x)
$$

if $\lambda= \pm \mu$.

Proof. It follows from (38) and (40) that

$$
\begin{aligned}
{\left[\mathrm{Si}_{+}(\lambda x) \circledast \sin (\mu x)\right]^{\prime}=} & \mu \mathrm{Si}_{+}(\lambda x) \circledast \cos (\mu x) \\
= & \frac{1}{4} \pi[\operatorname{sgn}(\lambda+\mu)+\operatorname{sgn}(\lambda-\mu)] \sin (\mu x) \\
& -\frac{1}{2} \ln \left|\frac{\lambda+\mu}{\lambda-\mu}\right| \cos (\mu x)
\end{aligned}
$$

and (50) follows. 
If $\lambda= \pm \mu$, it follows from (38) and (41) that

$$
\lambda \mathrm{Si}_{+}(\lambda x) \circledast \cos (\lambda x)=\frac{1}{4} \operatorname{sgn} \lambda \cdot \pi \sin (\lambda x)+\frac{1}{2}[c-\ln |2 \lambda|] \cos (\lambda x)
$$

and (51) follows.

COROLLARY 13. If $\lambda, \mu \neq 0$, then the neutrix convolutions $\mathrm{Si}_{-}(\lambda x) \circledast \sin (\mu x)$ and $\mathrm{Si}_{-}(\lambda x) \circledast \cos (\mu x)$ exist and

$$
\begin{aligned}
\mathrm{Si}_{-}(\lambda x) \circledast \sin (\mu x)= & -\frac{1}{4} \pi \mu^{-1}[\operatorname{sgn}(\lambda+\mu)+\operatorname{sgn}(\lambda-\mu)] \cos (\mu x) \\
& +\frac{1}{2} \mu^{-1} \ln \left|\frac{\lambda+\mu}{\lambda-\mu}\right| \sin (\mu x), \\
\mathrm{Si}_{-}(\lambda x) \circledast \cos (\mu x)= & \frac{1}{4} \pi \mu^{-1}[\operatorname{sgn}(\lambda+\mu)+\operatorname{sgn}(\lambda-\mu)] \sin (\mu x) \\
& +\frac{1}{2} \mu^{-1} \ln \left|\frac{\lambda+\mu}{\lambda-\mu}\right| \cos (\mu x)
\end{aligned}
$$

if $\lambda \neq \pm \mu$; and

$$
\begin{aligned}
& \mathrm{Si}_{-}(\lambda x) \circledast \sin (\lambda x)=\frac{1}{4} \lambda^{-1} \operatorname{sgn} \lambda \cdot \pi \cos (\lambda x)-\frac{1}{2} \lambda^{-1}[c-\ln |2 \lambda|] \sin (\lambda x), \\
& \mathrm{Si}_{-}(\lambda x) \circledast \cos (\lambda x)=\frac{1}{4} \lambda^{-1} \operatorname{sgn} \lambda \cdot \pi \sin (\lambda x)-\frac{1}{2} \lambda^{-1}[c-\ln |2 \lambda|] \cos (\lambda x)
\end{aligned}
$$

if $\lambda= \pm \mu$.

Proof. Equations (54) and (55) follow on replacing $x$ by $-x$ in (40) and (50), respectively; and (56) follow on replacing $x$ by $-x$ in (41) and (51), respectively.

The final neutrix convolutions follow easily from the above results:

$$
\operatorname{Si}(\lambda x) \circledast \sin (\mu x)=0, \quad \operatorname{Si}(\lambda x) \circledast \cos (\mu x)=0
$$

if $\lambda \neq \pm \mu$; and

$$
\operatorname{Si}(\lambda x) \circledast \sin (\lambda x)=0, \quad \operatorname{Si}(\lambda x) \circledast \cos (\lambda x)=0
$$

if $\lambda= \pm \mu$.

\section{REFERENCES}

[1] F. Al Sirehy and B. Fisher, On the sine integral, J. Nat. Sci. Math. 39 (1999), no. 2, 123-139.

[2] B. Fisher, Neutrices and the convolution of distributions, Univ. u Novom Sadu Zb. Rad. Prirod.-Mat. Fak. Ser. Mat. 17 (1987), no. 1, 119-135.

[3] B. Fisher and F. Al-Sirehy, On the cosine integral, Integral Transform. Spec. Funct. 8 (1999), no. 1-2, 31-42.

[4] B. Fisher and C. K. Li, On the cosine and sine integrals, Int. J. Appl. Math. 7 (2001), no. 4, 419-437.

[5] I. M. Gel'fand and G. E. Shilov, Generalized Functions. Vol. I: Properties and Operations, Academic Press, New York, 1964. 
[6] I. N. Sneddon, Special Functions of Mathematical Physics and Chemistry, Oliver and Boyd, London, 1956.

[7] J. G. Van der Corput, Introduction to the neutrix calculus, J. Analyse Math. 7 (1959/1960), 281-399.

Brian Fisher: Department of Mathematics AND Computer Sciences, Leicester UNIVERSITY, LEICESTER, LE1 7RH, ENGLAND

Current address: DePARTMENT of MATHEMATical SCIENCES, FACUlty of COMPUTER SCiences and Engineering, De Montfort University, The Gateway, Leicester, LE1 9BH, ENGLAND

E-mail address: fbr@1e.ac.uk

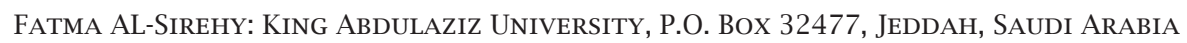

E-mail address: fatma2@emai1 .com 


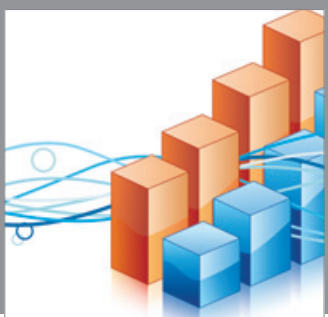

Advances in

Operations Research

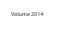

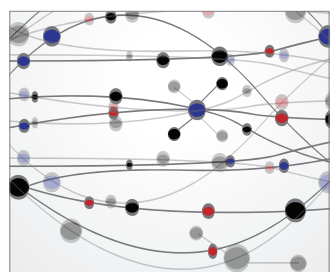

\section{The Scientific} World Journal
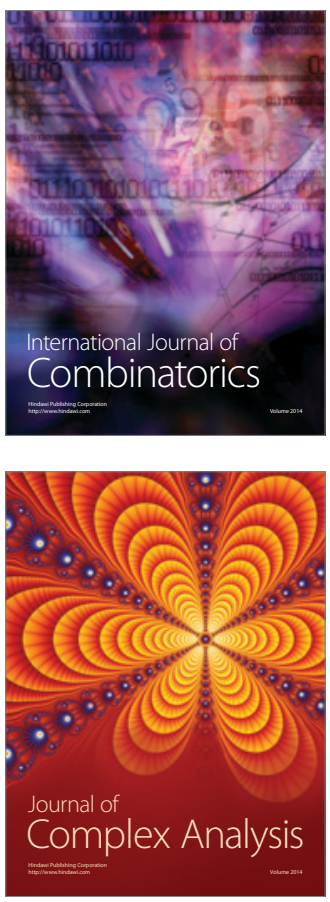

International Journal of

Mathematics and

Mathematical

Sciences
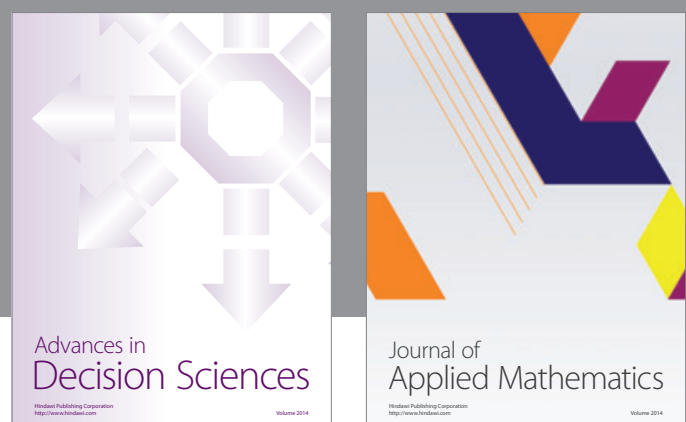

Journal of

Applied Mathematics
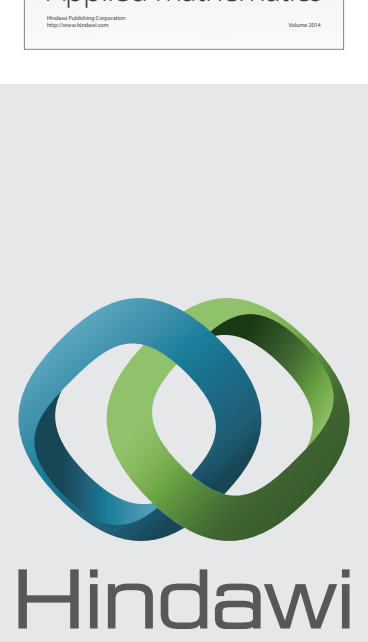

Submit your manuscripts at http://www.hindawi.com
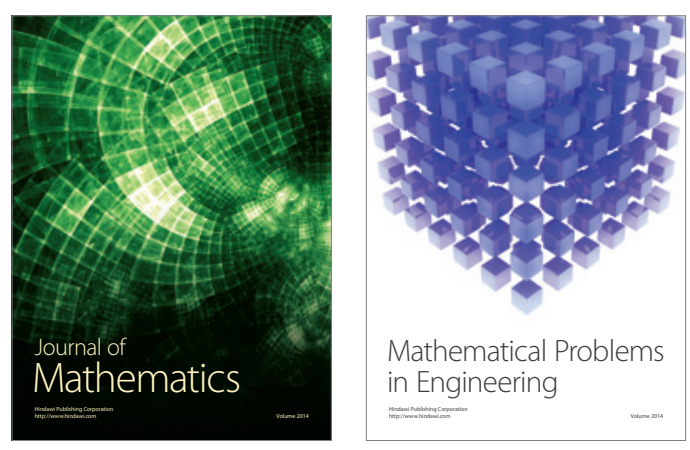

Mathematical Problems in Engineering
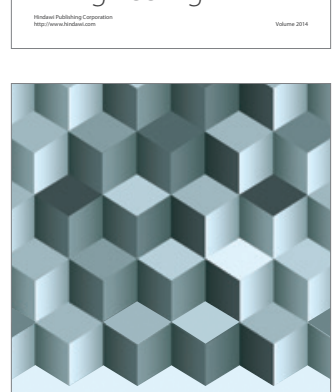

Journal of

Function Spaces
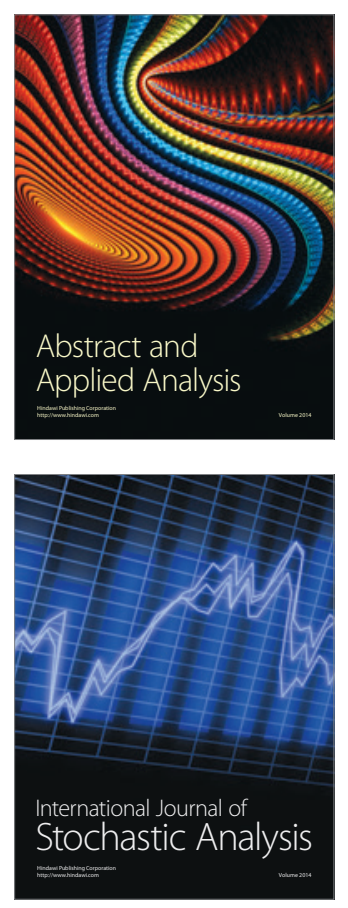

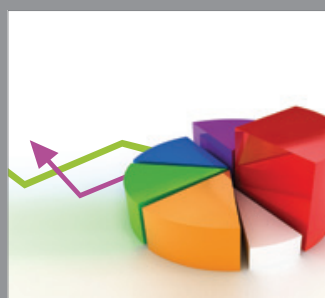

ournal of

Probability and Statistics

Promensencen
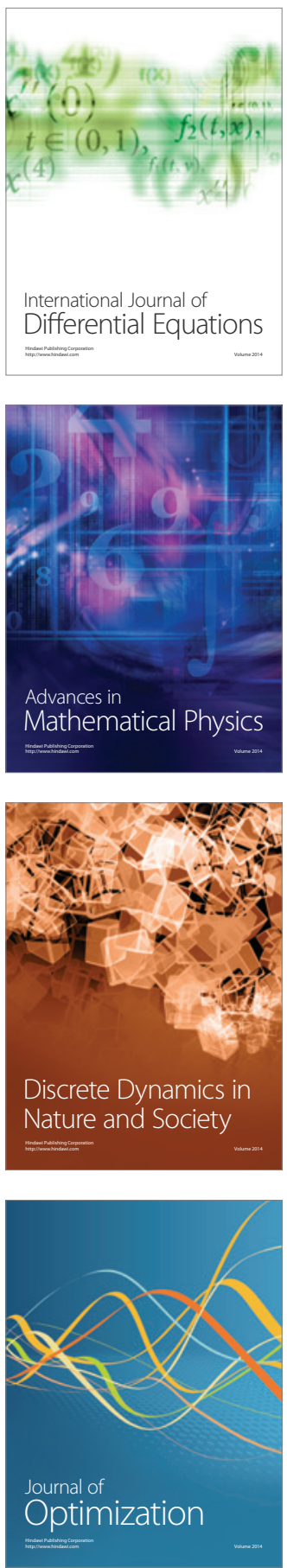